Maxted, W. R. (1957). J. gen. Microbiol. 16, 584-595

\title{
The Active Agent in Nascent Phage Lysis of Streptococci
}

\author{
BY W. R. MAXTED \\ Streptococcus Reference Laboratory, Public Health Laboratory Service, \\ Colindale, London, N.W. 9
}

SUMMARY : A powerful lytic factor has been obtained in phage lysates of group C streptococci, which is active against streptococci of groups $\mathrm{A}, \mathrm{C}$ and $\mathbf{E}$ and under some conditions group $\mathrm{H}$. It is the factor responsible for 'nascent' phage lysis. The lytic activity remains unaltered by the removal of the phage by high-speed centrifuging, and also in the presence of phage antiserum. It is active against young and old cell suspensions, live, or killed by chloroform. The activity diminishes in the absence of reducing agents and it is destroyed by proteolytic enzymes. Heat-killed cocci when attacked by the lytic factor become Gram-negative but do not lyse. The addition of a proteolytic enzyme completes lysis. Efforts to demonstrate the release of proteinases from streptococcal suspensions have failed. After lysis the group polysaccharide is free as a hapten and some cell-wall structure remains. $M$ antigen is also present in group A lysates.

In 1934 Evans reported that a phage, ordinarily lytic for group $\mathrm{C}$ but not for group A streptococci, lysed the group A cocci when sensitive group C streptococci were also present in the culture. No phage active on group $\mathbf{A}$ cocci could be recovered from the lysate. Evans considered that at the time of liberation the group $\mathrm{C}$ phage had a wider range of activity than later and referred to the phenomenon as 'nascent lysis'. This paper reports a re-investigation of the cause of nascent lysis, using the same phage as Evans. A powerful lytic agent was obtained from the lysates and this was studied to establish its origin and role in a 'nascent phage' system.

\section{METHODS}

The phage used was Evans's original phage B563, specifically active for group $\mathrm{C}$ streptococci. It proved to be very active on a stock group $\mathrm{C}$ strain Azgazardah (Griffith's original type 7) and this strain was used for propagation throughout.

Most of the group A strains employed were Griffith's original type strains kept lyophilized in this laboratory. Strains of other groups were from our stock collection originating from many sources.

The proteolytic enzymes used were: crystalline trypsin (Armour and Co. Ltd.); ficin and papain supplied in powder form (L. Light and Co. Ltd.); crystalline streptococcal proteinase generously supplied by Dr S. D. Elliott.

Phage propagation. A warmed $50 \mathrm{ml}$. volume of nutrient broth was seeded with $0.5 \mathrm{ml}$. of an $18 \mathrm{hr}$. broth culture of strain Azgazardah. After $2 \mathrm{hr}$. incubation, $1 \mathrm{ml}$. of stock phage suspension was added and incubation continued. The turbidity which had developed was cleared by phage action within $2 \frac{1}{2} \mathrm{hr}$. 
Nascent lysis. To $18 \mathrm{hr}$. broth cultures of the phage-resistant strain under investigation, $2-5 \%(\mathrm{v} / \mathrm{v})$ of an $18 \mathrm{hr}$. culture of the propagating strain was added. The mixture was used as an inoculum for broth or flooded over solid media to give a bacterial lawn. Lysis of the resistant strain upon the addition of phage to the mixture was termed 'nascent lysis'.

Testing of the lytic agent. Streptococcal cultures were concentrated by centrifugation so that approximately 3 drops from a $0.02 \mathrm{ml}$. dropping pipette suspended in $1 \mathrm{ml}$. of broth or phage lysate gave an optical density equal to an $18 \mathrm{hr}$. glucose broth culture, i.e. about $25 \times 10^{7}$ viable units $/ \mathrm{ml}$. A reducing agent, $0 \cdot 1 \mathrm{ml}$. of a $2 \%$ solution of neutralized thioglycollic (mercaptoacetic) acid, was added and the tubes placed in a water bath at $37^{\circ}$. The results, read by eye, were recorded as,,,+++++++++ , to denote the progressive decrease in opacity with complete clearing of the suspension as the end-point.

\section{RESULTS}

Most of Evans's work was performed with broth cultures, and she reported that phage B 563, specific in its filtered state for group $\mathrm{C}$ organisms, was lytic in its nascent state for all group $\mathbf{A}$ strains and for some members of group $\mathbf{E}$. In preliminary experiments in this laboratory the filtered phage could not be propagated on, or adapted to, any of the group A strains tested.

\section{Nascent phage reactions on solid media}

A large number of group A strains and some members of all the other Lancefield groups were tested on solid media for susceptibility to nascent lysis. Bacterial lawns were made with the test strain either alone or mixed with $5 \%(v / v)$ Azgazardah culture on nutrient agar containing $0.5 \%$ glucose and $5 \%(\mathrm{v} / \mathrm{v})$ horse serum. The stock phage was spotted on undiluted and in tenfold dilutions up to $10^{-5}$. All group $\mathbf{A}$ strains and both the group $\mathbf{E}$ strains tested were lysed when the group $\mathrm{C}$ cocci were present. Table 1 shows results representative of those obtained throughout.

\section{The fate of resistant group $\boldsymbol{A}$ strains during phage propagation}

The phage-propagating strain Azgazardah always gave a small compact colonial form on glucose serum agar, and could be readily distinguished from a strain of group A, type 6 (no. 8306) which formed large mucoid colonies on this medium. These two strains were incubated in broth separately and mixed, in both cases with and without phage. The six tubes were incubated and at intervals suitable dilutions were plated on serum glucose agar. After overnight incubation the group $\mathrm{A}$ and the group $\mathrm{C}$ colonies were counted (Fig. 1). Phage alone had no effect on the growth rate of the group $\mathbf{A}$ strain alone. In the mixture of groups A and C cocci, with phage present, both strains grew during the first hour and subsequently both were lysed but the numbers of group $\mathrm{C}$ organisms began to decrease first.

When the samples were quite clear they were tested for the presence of group polysaccharide by layering on groups $\mathbf{A}$ and $\mathbf{C}$ antisera. In the mixed 


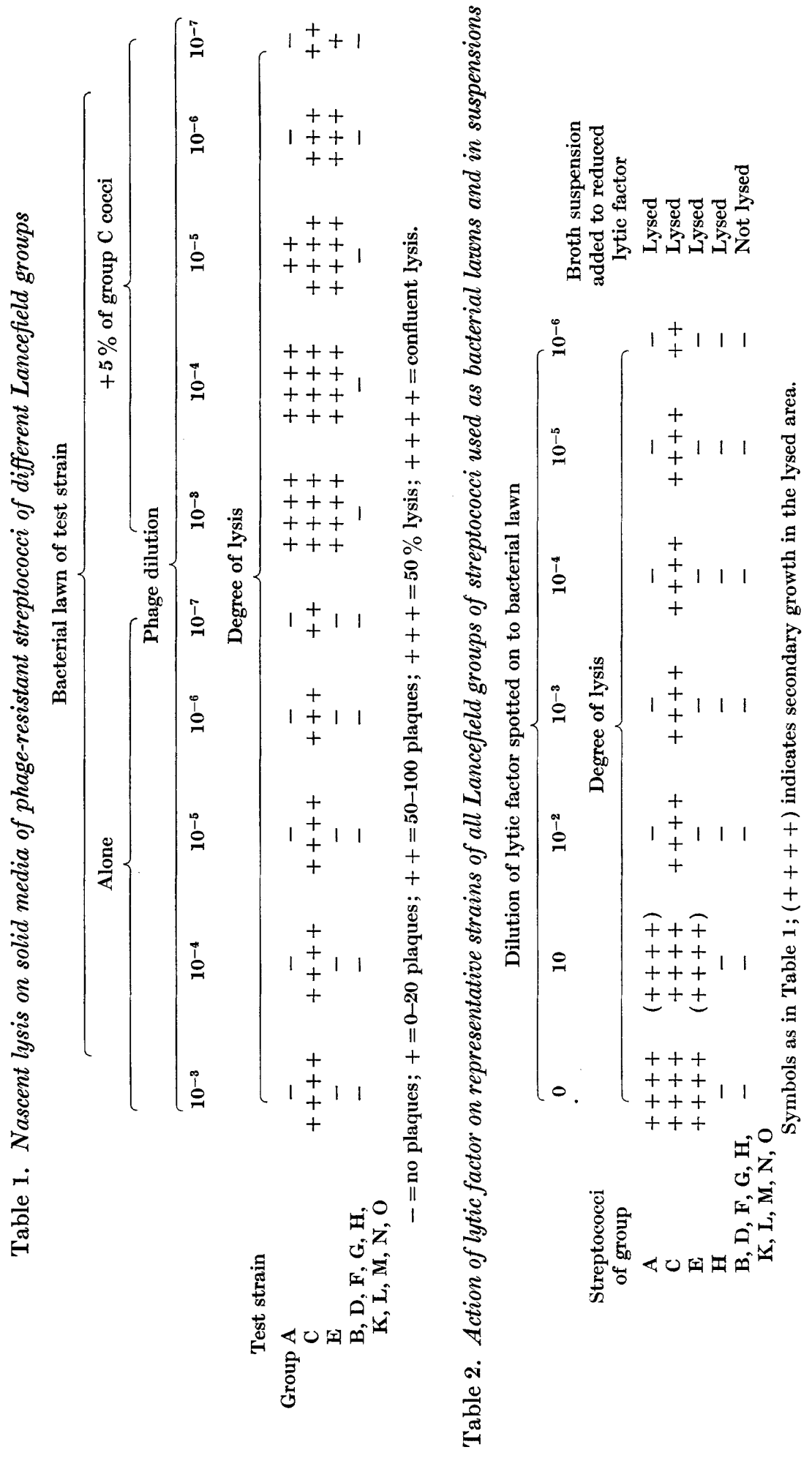


culture with phage, both group $\mathbf{A}$ and group $\mathrm{C}$ polysaccharides were present. The supernatant fluid of the control cultures, without phage, failed to react with antisera. There was therefore no doubt that the group $\mathbf{A}$ organisms were lysed and that the 'nascent phage' effect was not, as suggested by Whitehead, East \& McIntosh (1953) for the Streptococcus lactis phage system, the result of inhibition of the growth of the group A strain by large numbers of phage particles adsorbed on to each coccus. At the same time the lysate of the mixed culture, when filtered, contained phage active only on the group $\mathrm{C}$ strain. A second experiment with a group A type 24 strain gave a similar result.
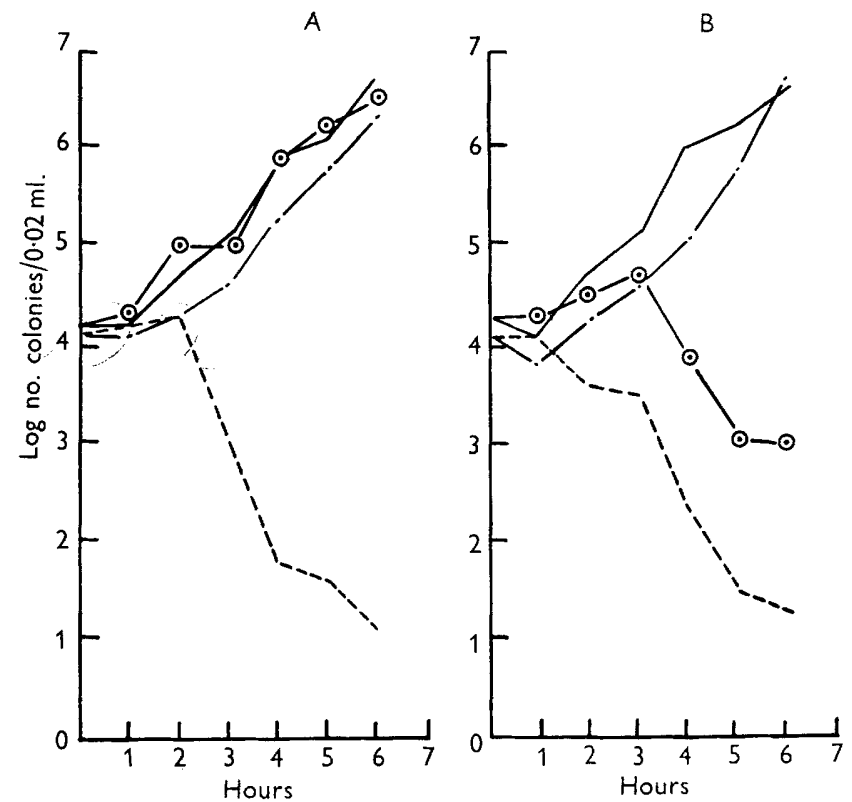

Fig. 1. Nascent lysis of group A streptococci in the presence of group $\mathbf{C}$ streptococci and group $\mathrm{C}$ phage, as shown by counts of viable units. Group A streptococci alone, group A streptococci + phage, $\odot-\odot$; group $\mathbf{C}$ streptococci alone, $-\cdot-$; group $\mathbf{C}$ streptococci + phage, - . - - . A. Both strains growing separately with or without phage. B. Both strains in the same culture with or without phage.

\section{A lytic factor in filtered nascent lysates}

The lysates produced during these experiments from the mixed culture and phage, and from the group $\mathrm{C}$ strain and phage, were later filtered and tested for lytic activity on group A cocci by adding a few drops of a well-grown culture of group A type 6 streptococci to $1 \mathrm{ml}$. of each filtrate and incubating in a water bath at $37^{\circ}$. Although the phage present was demonstrably not active on group $\mathbf{A}$ streptococcci there was, nevertheless, some lysis of the suspension in both samples after $2-3 \mathrm{hr}$. The addition of $0.1 \mathrm{ml}$. of a $2 \%$ solution of sodium thioglycollate increased this lytic activity considerably.

When a freshly propagated group $\mathrm{C}$ phage lysate was filtered and tested in the same way, rapid lysis of a heavy suspension of group A cocci occurred. 
This lytic power was a consistent feature of all the fresh group $\mathrm{C}$ phage filtrates, some of which could be diluted 1/500 and still show lytic activity against group A organisms. Undiluted samples lysed group A suspensions of $25 \times 10^{7}$ viable units $/ \mathrm{ml}$. in $2-3 \mathrm{~min}$. Reduction by thioglycollate was only necessary with old samples or with fresh samples when the content of organic material had been diluted during the process of partial purification.

\section{A lytic factor as the cause of nascent lysis}

If the nascent lysis as observed by Evans depended on the formation of the lytic factor described in the previous section it should be possible to obtain lysis with phage filtrate alone on all groups of streptococci which had shown nascent lysis when mixed with the phage and its propagating strain. Heavy suspensions of cocci of all groups used earlier were mixed with active samples of lytic factor, reduced with thioglycollate, and put into a water bath at $37^{\circ}$. Members of groups A, C and $\mathrm{E}$ lysed readily, while group $\mathbf{H}$ cleared more slowly. None of the other strains was affected.

A potent lytic-factor filtrate produced confluent lysis of a group A bacterial lawn when applied undiluted or at a dilution of 1/10 (Table 2). There was no effect at all in higher dilutions which should have given discrete plaques if such strong lysis were due to phage action. The 'nascent-phage' effect could thus be obtained using a good lytic factor lysate in the absence of the propagating strain.

\section{The role of the phage particle in nascent lysis}

Once formed the lytic factor did not require the presence of phage for its activity. Because the phage was inactive on group $\mathbf{A}$ cocci and the coccal suspension comparatively old, it seemed unlikely that the nascent lysis could be due to phage multiplication. An antiserum prepared against group $\mathbf{C}$ phage, when added to phage filtrates, completely neutralized the phage activity but did not affect the activity of the lytic factor. Phage could also be removed by high-speed centrifugation and samples containing a high concentration of lytic factor with relatively few phage particles were prepared by this means. The centrifuged deposit was taken up in a small amount of broth, thus giving a sample containing little lytic factor but a large amount of phage. All these preparations were reduced and tested for lytic activity against various suspensions of group A type 6 cocci. These suspensions were (1) a live suspension in the log phase, and (2) an $18 \mathrm{hr}$. broth culture tested $(a)$ untreated, $(b)$ killed with chloroform, $(c)$ killed by heating at $56^{\circ},(d)$ killed by heating at $85^{\circ}$ (Table 3).

The lytic factor was active against live and chloroform-killed suspensions and against cocci killed by heating to $56^{\circ}$ for $1 \mathrm{hr}$. Even when the phage content had been diminished to less than 1 particle/500 streptococci, lysis was still rapid. On the other hand, a heavy phage suspension containing little lytic factor was almost inactive. Cocci heated to $85^{\circ}$ were almost entirely resistant and subsequently it was found that group A streptococci killed by steaming for $1 \mathrm{hr}$. were completely resistant to lysis. In ditch plates the lytic factor 
diffused through the agar and remained lytic, but in a similar experiment the phage particles did not diffuse.

Table 3. The influence of age and viability on the susceptibility of group $A$ streptococci to lysis by the lytic factor with and without large numbers of phage particles present

Lysis produced by original lytic factor filtrate diluted

\section{Condition of group A culture}

In log. phase, alive $18 \mathrm{hr}$. culture, alive Chloroform killed

Heated to $56^{\circ}$

Heated to $85^{\circ}$

Phage titre

Condition of group A culture

In log. phase, alive $18 \mathrm{hr}$. culture, alive Chloroform killed

Heated to $56^{\circ}$

Heated to $85^{\circ}$

Phage titre

$\overbrace{1 / 2 \quad \text { Relative degree of lysis }}^{1 / 4} 1 / 8$

$\begin{array}{ccc}\overbrace{+++1++++} & ++++1++++ & +++1++++ \\ ++++1++++ & +++1++++ & +++1+++ \\ ++++1+++ & +++1++++ & +++1++++ \\ +1+ & +1+ & -1+ \\ \pm 1 \pm & \pm 1 \pm & -1 \pm\end{array}$

Lysis produced by supernatant after high-speed centrifugation diluted

$\overbrace{1 / 2}^{1 / 4} 1 / 8$

$$
\begin{array}{ccc} 
& \\
++++1++++ & ++++1++++ & ++++1++++ \\
++++1++++ & ++++1++++ & +++1+++ \\
++++1++++ & +++1++++ & +++1++++ \\
+/++ & +/++ & \pm /++ \\
\pm / \pm & \pm / \pm & \pm / \pm
\end{array}
$$

Lysis produced by phage deposit after high-speed centrifugation, resuspended in broth and diluted

\begin{tabular}{ccc}
\hline $1 / 2$ & Relative degree of lysis & \\
\hline $\pm 1++$ & $-1 \pm$ & $-1-$ \\
$\pm 1++$ & $-1 \pm$ & $-1-$ \\
$+1++$ & $+1++$ & $-1 \pm$ \\
$-1 \pm$ & $-1-$ & $-1-$ \\
$-1 \pm$ & $-1-$ & $-1-$
\end{tabular}

Condition of group A culture

In log. phase, alive 18 hr. culture, alive Chloroform killed Heated to $56^{\circ}$ Heated to $85^{\circ}$ Phage titre

The reading before the stroke was made after $15 \mathrm{~min}$. at $37^{\circ}$, and the one after the stroke after $45 \mathrm{~min}$. The symbols,,,++++++++++ denote the progressive decrease in the opacity of the suspension.

\section{Lysis of suspensions of heat-killed streptococci}

Group A streptococci which had been killed by steaming were not lysed by lytic-factor filtrate, although chloroform-killed organisms were susceptible. Heat-killed organisms were, however, lysed by filtrate when trypsin was added. The trypsin could be replaced by $0 \cdot 1 \%$ of ficin, papain or streptococcal proteinase. Trypsin, ficin and papain incubated alone with the lytic factor destroyed its activity. The heated cocci were therefore usually pretreated with the lytic factor and the proteolytic agent added after incubation for $10 \mathrm{~min}$. 
The results so far obtained with the lytic factor on heat-killed cocci suggested that streptococcal proteinase or intracellular autolytic enzymes were destroyed by heat. It might be that the lytic factor attacked the cell wall but failed to lyse the cell and that replacement of the streptococcal enzymes by a proteolytic enzyme was required for dissolution of the cell. If this were the case a proteolytic agent ought to be detectable in the lytic-factor lysates of streptococci or in streptococcal suspensions under suitable conditions.

Repeated attempts were made to demonstrate such a proteinase in group $\mathbf{A}$ culture supernatant (fresh and after standing for 1 week in the presence of the cocci at room temperature), in group A cocci disintegrated in a Mickle disintegrator and in lytic factor lysates of group A organisms. Substrate was also added to a mixture of group $\mathbf{A}$ cocci + lytic factor during actual lysis. Freshly propagated phage filtrate was also tested for proteolysis. The substrates employed were casein, gelatin, streptococcal $\mathbf{M}$ antigen, and heat-killed group A cocci pretreated with lytic factor. The tests were carried out at $\mathrm{pH}$ values of $7 \cdot 5,7 \cdot 0,5 \cdot 0$ and $3 \cdot 0$. A test for the streptococcal proteinase described by Elliott was carried out with an antiproteinase serum which gave a good precipitate when mixed with the enzyme. Culture supernatants of strain 8302 did not contain active streptococcal proteinase. None of these preliminary tests showed the presence of any proteinase. That the cocci of heat-killed suspensions were attacked to some degree by lytic factor was shown by the release of the group polysaccharide into the supernatant; they also became Gram-negative. This appeared to be the first visible change in suspensions of any susceptible organism but was never seen in cells resistant to lytic factor.

Electron-microscope preparations of heat-killed suspensions of group $\mathbf{A}$ streptococci treated with lytic factor showed some evidence of damage but the cytoplasmic mass remained dense and in coccoid form owing to the heating (Pl. 1, fig. 1). On the other hand, when live organism + lytic factor mixtures were examined, cocci apparently devoid of cytoplasm were seen among otherwise complete chains (Pl. 1, fig. 2) and later only cell envelopes remained, some with a little cytoplasm still retained ( $\mathrm{Pl}$. 1, fig. 3).

\section{Properties of the lytic factor}

The lytic factor was active over a $\mathrm{pH}$ range of $6 \cdot 5-8 \cdot 6$. Samples which were allowed to remain at a $\mathrm{pH}$ value below 4.0 or above 8.6 for some hours, and then readjusted to $\mathrm{pH} 7 \cdot 5$, were inactive. That the lytic factor was a protein was suggested by its destruction by proteinase. It was precipitated by $40 \%$ saturation with ammonium sulphate; subsequent dialysis against tap water left a weak product which often increased in potency on the addition of ammonium sulphate. The crude lytic material could be freeze-dried without loss of activity.

It has not so far been possible to prepare an antiserum which neutralizes the lytic factor; all attempts have resulted in sera which neutralize the phage but completely fail to neutralize the lytic factor. Phage was always present in the material used as an antigen, and the inoculation of rabbits with the lytic-factor filtrate in which the phage was neutralized by antiphage serum also stimulated the production of antibodies to the phage. 


\section{The production of the lytic factor}

The strength of the lytic factor produced during phage propagation seemed to depend on the amount of phage multiplication. Five propagations were made with a standard number of phage particles added to a varying number of organisms. A $50 \mathrm{ml}$. volume of warmed broth was seeded with a heavy inoculum of group $\mathrm{C}$ cocci and incubated for $4 \mathrm{hr}$. at $37^{\circ}$. A viable count was made and the culture then diluted $1 / 2,1 / 5,1 / 25$ and 1/125 in broth. To $10 \mathrm{ml}$. volumes of each dilution and of the undiluted cultures $0.4 \mathrm{ml}$. of phage stock was added and the cultures incubated for $2 \mathrm{hr}$. All except the undiluted sample were quite clear at this time, and this sample cleared after being kept at $4^{\circ}$ overnight. All samples (stored overnight at $4^{\circ}$ ) were then tested for phage titre and for the potency of the lytic factor. The phage concentration and the lytic activity were greatest in the $1 / 2$ sample which had had $1 \cdot 3 \times 10^{8}$ viable units $/ \mathrm{ml}$. and an initial bacterium : phage ratio of 13.7:1 (Table 4). The sample in which there had been the heaviest concentration of organisms and which had lysed rather slowly during the propagation period had a poor phage titre, and although very large numbers of cocci had lysed ultimately, the potency of the lytic factor was also very low.

In another experiment a constant number of cocci in the logarithmic growth phase were mixed with different concentrations of phage and the lytic factor activity and final phage content estimated. Although the initial bacterium : phage ratio ranged from $2 \cdot 5: 1$ to $0 \cdot 1: 1$, the lytic factor activity was approximately equal in all mixtures. The final concentration of phage particles in each sample was also equal (Table 5).

The conclusion reached from a number of experiments of a similar type was that the lytic factor was strongest when phage propagation was best and that this occurred where the number of propagating cocci was relatively large, with multiplicity of phage infection of 4 . The lytic factor appeared soon after the beginning of lysis and was never present in streptococcal cultures or disintegrates, unless phage lysis had taken place.

It seemed that at least a part of the lytic factor was produced by phage or by the streptococci under the influence of phage and was released at the time of release of phage. If it had been a product of lysed cocci alone it might have been possible to have built up the activity of a poor lytic-factor sample, from which phage had been removed, by the addition of streptococci at intervals as lysis was completed. The activity of the lytic factor was not increased by this procedure.

Dubos (1937) obtained from autolysing pneumococci an enzyme capable of causing pneumococci to become Gram-negative and Pirie (1939), with Escherichia coli, showed an enzyme present in culture supernatant fluids which attacked the coli bacterial polysaccharide. Efforts to find such an enzyme in cultures of the group $\mathbf{C}$ strain Azgazardah were unsuccessful.

In considering the source of the lytic factor it seemed worth while testing a number of group $\mathrm{C}$ strains for propagation of the phage to find whether the lytic factor was always produced when phage B 563 multiplied, or whether it 


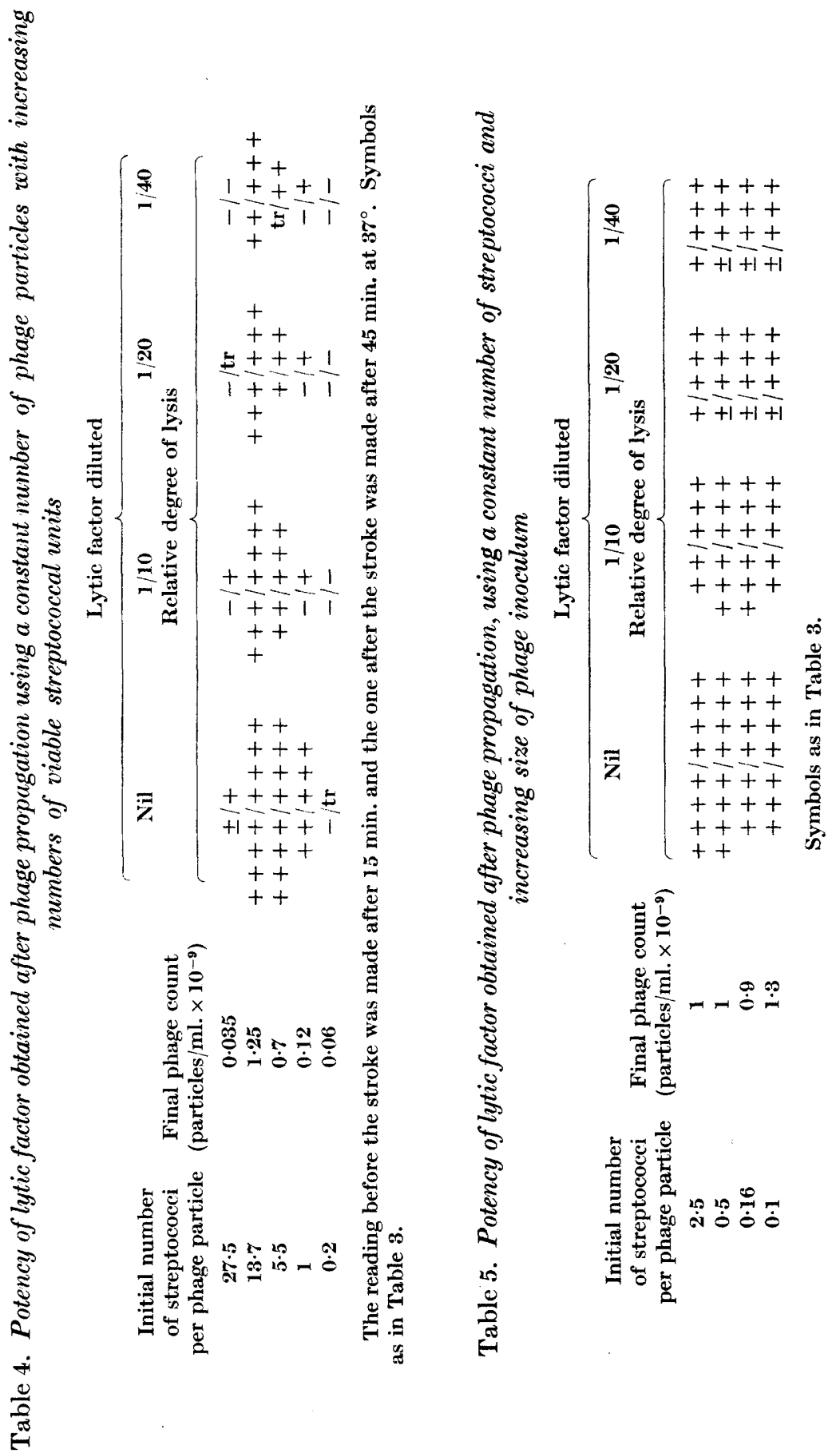


was peculiar to its multiplication on the strain Azgazardah. From a large range of group C strains, 12 were selected as representing those whose characteristics of haemolysis, colony form, and host range, showed the greatest diversity. The phage was eventually adapted to all the strains, although it propagated much more successfully on some than on others. In each case active lytic factor was produced and the strength of the samples ran parallel with the number of phage particles liberated.

With the phage used, therefore, the lytic factor was produced from all susceptible streptococci. To test the effect of different phages on the one organism repeated attempts were made to obtain phages from some twenty group C strains and three samples of sewage, but none was isolated. However, another group $\mathrm{C}$ phage, originating from sewage and supplied by Dr Asheshov, also produced the lytic factor during propagation on a group $\mathrm{C}$, strain 6 , supplied with it. The stock phage B 563 used in all the other experiments also produced the lytic factor when propagated on strain 6 . The two phages were both lytic phages and were both neutralized by antiserum with $\mathrm{B} 563$. The two propagating strains, however, were different: Azgazardah was of human origin, while strain 6 was an animal strain.

\section{DISCUSSION}

The extremely active lytic agent described here is present in lysates of the group C streptococcus strain Azgazardah produced by the action of a lytic phage. This agent is capable of lysing live or chloroform-killed suspensions of streptococci of groups $\mathrm{A}, \mathrm{C}$ and $\mathrm{E}$, and it seems likely that the agent is responsible for the nascent lysis observed by Evans on streptococci belonging to these groups. Virulent phages active against group $\mathbf{A}$ streptococci and used in previous work (Maxted, 1955) also gave nascent lysis of otherwise resistant members of group $\mathbf{A}$; such phage propagations also yielded a lytic principle similar to the one described here, although so far they have always been much less potent. This would agree with the observations of Evans that phage B563 was by far the most active of her phages in respect of nascent lysis.

It is not yet clear whether the lytic factor is produced by the phage alone or whether it is a streptococcal product activated by the phage, or a streptococcal product that activates some substance liberated by the phage; it is, however, only demonstrable when phage lysis has taken place. A very large number of cocci could be lysed with a low multiplicity of phage infection, but unless the phage propagation was good the lytic factor potency was poor. It seems to be similar to the substance obtained by Wahl \& Josse-Goichot (1950) from Streptococcus lactis. They suggested that their agent was a product of the streptococcus since the greater the proportions of organisms to phage in the propagation, the stronger the lytic agent. Weidel (1953) suggested that some phages may be a good source of cell-wall enzymes.

A dramatic characteristic of the lytic factor is the speed with which a potent sample will lyse comparatively heavy suspensions of live group A cocci. Lysis 
of phage-resistant organisms by this agent is certainly independent of the phage particle and is unlike some similar agents found by others in phage lysates of various other species.

A number of lysins have been described for Gram-negative bacilli, such as the lysin B of Sertic (1937) which destroys some surface antigens but does not lyse the bacilli, and the prolysins of Panijel \& Huppert (1956) which lyse acetone treated bacteria but not living organisms growing in broth. Ralston, Baer, Lieberman \& Krueger (1955) described a lytic principle, 'virolysin', found in phage lysates of some staphylococci, which could lyse staphylococci killed by heating to $56^{\circ}$ but could only lyse live staphylococci in the logarithmic phase of growth when phage particles were present. On the other hand, the present streptococcal lytic factor readily lyses young live cultures but is much less active on heated suspensions and requires the addition of proteinase to complete the lysis of suspensions which have been heated above $80^{\circ}$. Pursuing an observation made by $\mathrm{Dr} \mathrm{Kjems}$ of Copenhagen (private communication, 1955 ) it has been possible to demonstrate a lytic agent present in phage lysates of enterococci which is similar to 'virolysin', and differs from the lytic factor in the same way as 'virolysin' in its action on heat-killed organisms.

McCarty (1952) studied the lytic substances in the streptomyces filtrate that has been used to lyse haemolytic streptococci (Maxted, 1948) and suggested that there are two enzymes. One of these initiates the process by attacking the cell wall and thus prepares the cell for complete dissolution by the second, a proteolytic enzyme. There is an apparent analogy between the streptomyces lytic factor and the one described here, which shows a similar need for an added proteolytic factor in order to lyse heat-killed cocci. But it may be that with living streptococci no proteolytic activity is involved; certainly at present there is no evidence for major protein destruction. The great increase in the protein content in lytic factor lysates of streptococci, as shown by precipitation with trichloroacetic acid, suggests that after the initial attack on the cell wall or cell membrane the cytoplasmic material diffuses into the surrounding fluid. The cell walls of heated streptococci are also attacked, but the cytoplasmic material appears to remain intact perhaps because of the alteration of its physical state by heat. Preliminary electron microscope examination supports this view.

A single lytic agent of biological origin capable of lysing fast-growing streptococci, with so little apparent destruction of cell contents, should prove of some interest. It may prove a useful tool in the study of streptococci and it points at once to a difference between the structure or composition of groups A, C and E strains and those of other groups; it also offers a quick and simple means of obtaining intracellular elements. The use of purified lytic factor and purified cell-wall material prepared by mechanical means may disclose the site of attack and the part played in lysis by proteolytic agents. Work on this aspect is in hand.

The phage B 563 used throughout this work is a virulent phage. There are possibly three kinds of phage active on group $\mathbf{A}$ streptococci: the virulent phages originating from sewage, temperate ones reported by Kjems (1955), and 
Journal of General Microbiology, Vol. 16, No. 3

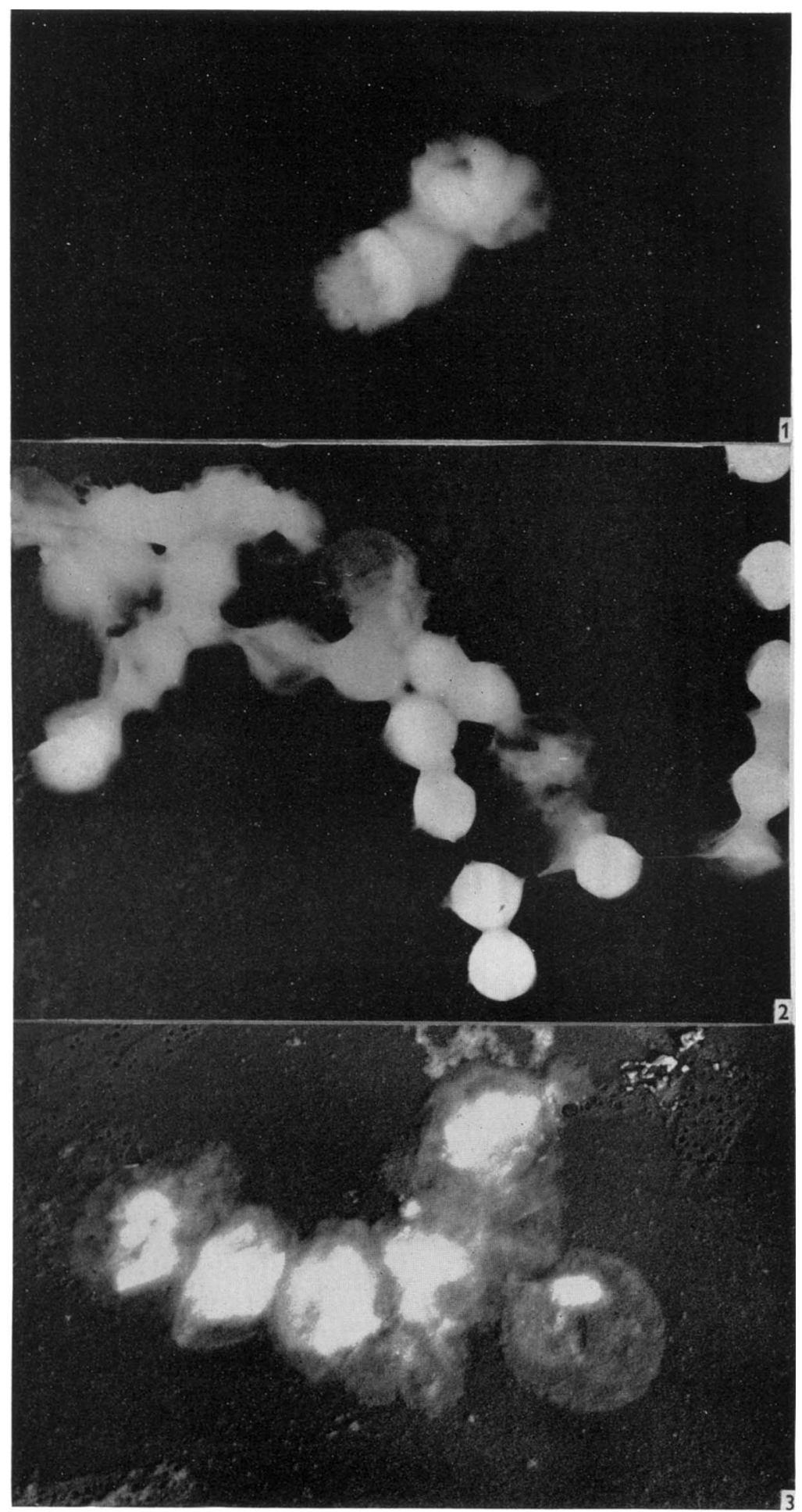

W. R. Maxted-Naschint phage i.ysis. Puate 1. 
other temperate phages isolated here (Maxted, in preparation). There is, however, reason to believe that not all produce a lytic factor similar to the one described here and it may be that this phenomenon is a feature of the virulent streptococcal phages only.

My thanks are due to Dr R. C. Valentine of the National Institute for Medical Research for the electron micrographs, and for guidance in their interpretation.

\section{REFERENCES}

Dubos, R. J. (1937). The autolytic system of pneumococci. J. exp. Med. 65, 873. Evans, A. C. (1934). Streptococcus bacteriophage: a study of four serological types. U.S. Publ. Hlth Rep. 49, 1386.

KJems, E. (1955). Studies on streptococcal bacteriophages. Acta path. microbiol. scand. 34, 433.

MaXted, W. R. (1948). Preparation of extracts for Lancefield grouping. Lancet, ii, 255.

MaXted, W. R. (1955). The influence of bacteriophage on Streptococcus pyogenes. J. gen. Microbiol. 12, 484.

McCarty, M. (1952). The lysis of group A hemolytic streptococei by extracellular enzymes of Streptomyces albus. I. Production and fractionation of the lytic envymes. J. exp. Med. 96, 555 .

PaniJel, J. \& Huppert, J. (1956). Recherches sur les prolysines. I. La prolysine du phage $\mathrm{F}^{\mathrm{C}} \mathrm{Z}$. Ann. Inst. Pasteur, 90, 619.

Pirie, A. (1939). A chemical change occurring during the lysis of Bact. coli by bacteriophage. Brit. J. exp. Path. 20, 99.

Ralston, D. J., Bafr, B., Lieberman, M. \& Krueger, A. P. (1955). Virolysin: a virus induced lysin from staphylococcal phage lysates. Proc. Soc. exp. Biol., N.Y. 89, 502 .

Sertic, V. (1937). Sur le rôle de la lysine B au cours de la bactériophagie. C.R. Soc. Biol., Paris, 126, 1074.

WAHL, R. \& JosSE-GoIcrot, J. (1950). Le facteur bactériostatique des lysats bactériophagiques. C.R. Acad. Sci., Paris, 230, 1703.

Weides, W. (1953). Further studies on the membrane of ' $E$. coli' B. Ann. Inst. Pasteur, 84, 60.

Whitehead, H. R., EAst, A. \& McIntosh, L. (1953). The so-called nascent bacteriophage phenomenon. J. Dairy Res. 20, 60.

\section{EXPLANATION OF PLATE}

Electron micrographs; all preparations were fixed in osmium tetroxide; platinum shadowed.

Fig. 1. Appearance of heat-killed streptococci after treatment with lytic factor. $\times \mathbf{2 5 , 0 0 0}$.

Fig. 2. First stage in lysis of live streptococci by dilute lytic factor. $\times 15,000$.

Fig. 3. Residual structure remaining after fast lysis of living streptococei has taken place. $\times 30,000$. 